\title{
Animal Naming Test - a simple and accurate test for diagnosis of minimal hepatic encephalopathy and prediction of overt hepatic encephalopathy
}

\author{
Ankit Agarwal, Sunil Taneja, Madhu Chopra, Ajay Duseja, Radha K Dhiman \\ Postgraduate Institute of Medical Education and Research, Chandigarh, India
}

\begin{abstract}
Aim of the study: Minimal hepatic encephalopathy (MHE) is the mildest form in the spectrum of hepatic encephalopathy (HE). We compared the usefulness of the Psychometric Hepatic Encephalopathy Score (PHES) and Animal Naming Test (ANT) for the diagnosis of MHE and the prediction of the development of overt episodes of HE.

Material and methods: 103 consecutive patients with liver cirrhosis without overt HE were subjected to PHES and ANT evaluation. The receiver-operating characteristic curve was used to determine the optimum cut-off of the ANT value for the diagnosis of MHE.

Results: Thirty-seven (35.9\%) patients had MHE as assessed by altered PHES. ANT $(<14)$ was positive in $36(34.95 \%)$ patients with MHE with a sensitivity of $89.19 \%$ and specificity of $95.7 \%$, positive predictive value (PPV) of $91.67 \%$, negative predictive value (NPV) of $94.03 \%$ and diagnostic accuracy of $93.20 \%$. The area under the curve for diagnosis of MHE was 0.978 (95\% Cl: 0.954-1.0). MHE patients had significantly lower ANT as compared to non-MHE patients and controls $(10.81 \pm 0.324$ vs. $15.27 \pm 0.147$ vs. $15.78 \pm 0.192$, respectively, $p=0.01)$. ANT correlated with PHES $(r=0.752, p=0.001)$ and also with Child-Pugh $(r=-0.408, p=0.001)$ and MELD $(r=-0.318, p=0.001)$ scores. During follow-up, 14 patients in the MHE group and 4 in the nonMHE group developed overt episodes of HE $(p=0.001)$.

Conclusions: ANT is simple and accurate for the diagnosis of MHE and prediction of overt episodes of HE in patients with cirrhosis and correlates well with the Child-Pugh and MELD scores.
\end{abstract}

Key words: minimal hepatic encephalopathy, hepatic encephalopathy, cirrhosis.

Address for correspondence:

Assist. Prof. Sunil Taneja, Department of Hepatology, Postgraduate Institute of Medical Education and Research, Chandigarh 160012, India, phone: +91 9592160444, e-mail: drsuniltaneja@hotmail.com

\section{Introduction}

Hepatic encephalopathy (HE) is characterized by a range of neuropsychiatric symptoms that occur in patients with chronic liver disease after the exclusion of already known neurological disorders [1,2]. Minimal hepatic encephalopathy (MHE) is a comparably low degree of cognitive impairment in patients having cirrhosis of the liver and is regarded at one end of the spectrum of $\mathrm{HE}$ rather than being a distinct entity $[3,4]$.

The present diagnostic criteria of MHE rely on the patient's history, exclusion of other neurological diseases, detailed physical examination, and the results of neurophysiological and neuropsychological tests $[5,6]$. As there is no gold standard, most trusted methods that are frequently used to diagnose MHE include psychometric and neurophysiological tests $[3,7]$. However, simple tests are needed for diagnosis of MHE given its sociomedical relevance. A standard simple verbal questionnaire was applied in a recent study in Italy for the rapid assessment of patients who do not have HE with frank disorientation. This Animal Naming Test (ANT) score was found to be accurate and directly correlated with Psychometric Hepatic Encephalopathy Score (PHES) as well as with different stages of HE. 
Table 1. Clinical and demographic features of patients enrolled in the study

\begin{tabular}{|c|c|c|c|}
\hline Parameter & $\begin{array}{l}\text { Patients enrolled } \\
\qquad(n=103)\end{array}$ & $\begin{array}{c}\text { MHE }^{\mathrm{a}} \\
(n=37)\end{array}$ & $\begin{array}{l}\text { NMHE }^{a} \\
(n=66)\end{array}$ \\
\hline Sex $\left(\right.$ male) ${ }^{b}$ & $86(83.5 \%)$ & $31(83.8 \%)$ & $55(83.3 \%)$ \\
\hline Age (years) ${ }^{c}$ & $48.029(45.87-50.18)$ & 48.027 (44.907-51.147) & $48.030(45.105-50.956)$ \\
\hline \multicolumn{4}{|l|}{ 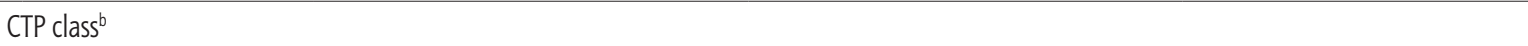 } \\
\hline Class A & $61(59.22 \%)$ & $12(19.7 \%)$ & $49(80.3 \%)$ \\
\hline Class B & $27(26.21 \%)$ & $14(51.9 \%)$ & $13(48.1 \%)$ \\
\hline Class C & $15(14.5 \%)$ & $11(73.3 \%)$ & $4(26.7 \%)$ \\
\hline MELD score ${ }^{c}$ & 13.74 (12.90-14.58) & 16.19 (14.79-17.58) & $12.36(11.44-13.28)$ \\
\hline \multicolumn{4}{|l|}{ Etiology } \\
\hline Alcohol & $57(55.3 \%)$ & $23(62.1 \%)$ & $34(51.51 \%)$ \\
\hline HBV & $11(10.7 \%)$ & $4(10.8 \%)$ & $7(10.6 \%)$ \\
\hline $\mathrm{HCV}$ & $9(8.7 \%)$ & $3(8.1 \%)$ & $6(9.09 \%)$ \\
\hline Others & $26(25.3 \%)^{d}$ & $7(18.9 \%)$ & $19(28.78 \%)$ \\
\hline Education in years ${ }^{c}$ & 11.223 (10.74-11.77) & 10.595 (9.926-11.264) & 11.576 (10.938-12.214) \\
\hline
\end{tabular}

aMHE and NMHE columns describe patients who have been enrolled; the distinction between the 2 groups was based on the results of the Psychometric Hepatic Encephalopathy (PHES) score. ${ }^{b}$ Number (percentage with 95\% confidence interval); ${ }^{*}$ Mean (95\% confidence interval); ${ }^{d}$ Non-alcoholic steatohepatitis 10 patients, Budd Chiari syndrome 1 , and cryptogenic cirrhosis in 15 patients.

Therefore, the present study was conducted to compare the prevalence of PHES, and ANT abnormalities in patients having cirrhosis without any evidence of overt hepatic encephalopathy (OHE) and to study the relationships of these abnormalities with the severity of liver disease and the development of OHE and survival on follow-up in an Indian population.

\section{Material and methods}

The Ethics Committee of the Postgraduate Institute of Medical Education and Research (PGIMER), a tertiary level health care hospital in Chandigarh, India, approved the study protocol (Reference No: NK/3725/ $\mathrm{MD} / 566)$. Each subject gave written informed consent before inclusion in the study. The guidelines laid down by the Indian Council of Medical Research (1994) and the Helsinki Declarations (modified 1989) were adhered to in all patients in the study. A cross-sectional and prospective study was utilized.

\section{Patient selection}

One hundred and twenty-three patients with cirrhosis of the liver without evidence of OHE who attended the outpatient Liver Clinic of the Department of Hepatology, Postgraduate Institute of Medical Education and Research, Chandigarh, were candidates for enrolment; 103 patients were included in the study and 20 patients were excluded as they fulfilled either one or more of the exclusion criteria (Table 1, Fig. 1). The diagnosis of cirrhosis of the liver was based on clinical, biochemical, and ultrasonographical, and/or liver histological data. The etiological workup of cirrhosis including alcohol, chronic hepatitis $\mathrm{B}$ and $\mathrm{C}$, autoimmune hepatitis, primary biliary cirrhosis, primary sclerosing cholangitis, non-alcoholic steatohepatitis, and Wilsons disease was performed as described in our previous study [1].

\section{Controls}

Fifty healthy volunteers mostly including friends or family members of the patient and hospital workers

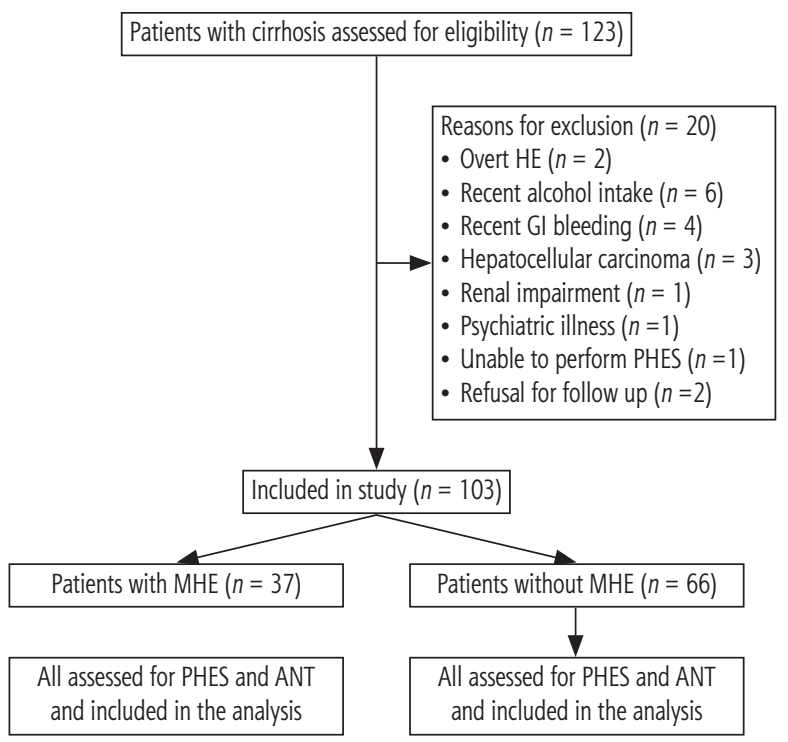

Fig. 1. Flow chart of patients included in study 
Table 2. Comparison between controls and cirrhotic patients

\begin{tabular}{lccc}
\hline Parameter & $\begin{array}{c}\text { Controls } \\
(\boldsymbol{n}=50)\end{array}$ & $\begin{array}{c}\text { Cirrhotics } \\
(\boldsymbol{n}=103)\end{array}$ & P-value \\
\hline Age (years) & $\begin{array}{c}45.820 \\
(43.907-48.105)\end{array}$ & $\begin{array}{c}48.029 \\
(45.87-50.18)\end{array}$ & 0.194 \\
\hline Sex (M/F) $)^{\dagger}$ & $86 / 17$ & $41 / 09$ & 0.817 \\
\hline $\begin{array}{l}\text { Occupation } \\
\text { (blue/white collar) })^{\dagger}\end{array}$ & $57 / 46$ & $31 / 19$ & 0.434 \\
\hline Education (years) & 11.340 & 11.223 & 0.591 \\
\hline ANT > 14 & $(11.97-13.06)$ & $(10.74-11.77)$ & \\
\hline ANT 10-13 & $48(96.0 \%)$ & $67(65.1 \%)$ & \\
\hline ANT < 10 & $2(4.0 \%)$ & $25(24.3 \%)$ & \\
\hline
\end{tabular}

ANT - Animal Naming Test, M- male, F-female, ${ }^{\dagger}$ Number; ${ }^{*}$ mean $195 \%$ confidence interval).

which included paramedical staff and technicians were enrolled as controls in the study. Neither the patient nor the control subjects were on any psychometric performance-altering drugs, i.e., benzodiazepines, anti-epileptics, or psychotropic agents. There was no evidence of neurological or liver disease or psychiatric ailment in the controls. The controls undertook only ANT testing for the determination of normative cut-offs in the Indian population. Since PHES has earlier been validated in the Indian population, controls did not undergo PHES testing and the cut-off for PHES as determined earlier was used [2]. The details of patients and their comparison with the control subjects are shown in Table 2 .

\section{Exclusion criteria}

The exclusion criteria for the study were previous episodes of overt HE ( $\leq 3$ months), history of alcohol use ( $\leq 3$ months), gastrointestinal bleeding or antibiotic use ( $\leq 6$ weeks), use of drugs impairing psychometric performances ( $\leq 6$ weeks), any history of shunt surgery, renal impairment or electrolyte imbalance or patients having hepatocellular carcinoma and other medical diseases such as pulmonary disease, heart failure, psychiatric or neurological disorders.

\section{Clinical and laboratory assessments}

The clinical examination included general physical, systemic and neurological examination. The Mini-Mental State Examination (MMSE) was performed in all patients to rule out the presence of any concomitant illness that may influence the quality of life or the neurological status of the patient. The mental state of patients suffering from cirrhosis was divided into grade 0 and grade $1 \mathrm{HE}$ based on West Haven criteria. The labora- tory investigations included complete biochemical and hematological profile and a coagulogram. An upper GI endoscopy was done in all the patients for the detection of varices. The severity of liver disease was assessed using Model of End-Stage Liver Disease (MELD) and Child-Turcotte-Pugh (CTP) scores.

\section{Neuropsychological assessment}

The PHES has been extensively validated in German [8], Indian [2], Spanish [9], Italian [10] and Romanian [11] populations. It can be performed in 10-15 minutes in an outpatient department [2]. The PHES consists of a battery of six tests including the number connection test NCT-A, the NCT-B, digit symbol test, serial dotting test, and the line tracing test for time (t) and error (e). Due to concerns about some patients who are not acquainted with English alphabets and not able to perform NCT-B, it was replaced by the figure connection test (FCT-A) [2]. The FCT and the NCT are similar in principle, but the numbers are substituted by figures. FCT is used to assess the mental state and it surpasses the hurdles of the differences in language and the level of education and literacy of the person. The clinical impact of the FCT has already been assessed in large numbers of patients with MHE [12]. A PHES score of greater than or equal to -5 is considered diagnostic of MHE in the Indian patients.

\section{Animal Naming Test}

The Animal Naming Test (ANT) is an analysis of semantic fluency consisting of saying as many animal names as possible within one minute. It is a verbal questionnaire to obtain a rapid assessment of patients who have cirrhosis either in an office setting or at the bedside. It is an easily obtainable measure of cognition like the Glasgow Coma Scale (GCS) for patients with coma or questions regarding orientation in time, space and identity. In a well-lit room with quiet surroundings not having any obvious external disturbances, the patient is asked to speak out the names of as many animals as possible in 1 minute and responses are recorded. If the patient stops before 1 minute, he is asked about any more animals he would like to add. If the patient does not speak for 15 seconds, he is given a hint; e.g. a lion is an animal, can he/she name any more animals? After 1 minute, all the responses are counted excluding repetitions and non-animal words.

The optimal performance of ANT requires wellorganized verbal recall and retrieval, along with self-monitoring of cognition (the patient should keep track of the name of animals already spoken), with 
inhibition of responses, when required. These cognitive skills require adequate memory as well as effective executive functions. The ANT is very sensitive to functions associated with anterior cortical and prefrontal cortex areas. These parts of the brain are particularly sensitive to the initial stages of HE. Therefore, ANT can be used as a first-line test for a patient with cirrhosis for the evaluation of HE. Humans of every culture are reasonably well knowledgeable in the names of animals, and hence the influence of gender, age and education, if any, may be limited.

\section{Follow-up}

At least 6 months follow-up was done for each patient for the external validity of the ANT. The endpoint was the development of OHE and survival.

\section{Statistical analysis}

Data were expressed as mean with $95 \%$ confidence interval (CI) and proportions with 95\% CI where appropriate. Neuropsychological assessment by PHES and ANT was done in all patients with cirrhosis of the liver, which was correlated amongst patients in the two groups, i.e. (i) without MHE and (ii) with MHE. For normally distributed data, ANOVA was used and for skewed data the Kruskal-Wallis test followed by the Mann-Whitney test was used. Statistical analyses for categorical data were performed using the $\chi^{2}$ test or Fisher's exact test. The cut-off value of ANT for the diagnosis of MHE was determined using ROC curves. The relationships between neuropsychological tests or PHES and ANT were assessed by Spearman's rank correlation coefficient rho. A multivariate logistic regression analysis using the block method was performed on variables reaching a significance of $p<0.05$ on univariate analysis to determine their influence on the presence of MHE. The risks estimated from the Cox regression models were expressed as the hazard ratio (HR) with their respective $95 \%$ CI. The cumulative probability of death, as well as the incidence of OHE in patients, was analyzed using the Kaplan-Meier method. The logrank test was used to compare Kaplan-Meier survival curves. A probability level of $p<0.05$ was set for statistical significance. Statistical analysis was performed with SPSS software for Windows, IBM SPSS statistics version 22.0.

\section{Results}

Between July 1, 2017, and June 30, 2018, 123 patients with cirrhosis of the liver were screened. Out of these, 103 patients $(83.7 \%)$ met the criteria for eligibility and were included in the study. Figure 1 shows the number of patients enrolled in the study and the reason for excluding 20 patients (16.2\%). The clinical and demographic features of the enrolled patients are shown in Table 1.

Of the 103 patients 86 men and 17 women were included in the study. The etiology of liver cirrhosis was as follows: patients with alcohol abuse 57 (including hepatitis $B$ and alcohol, 3 patients: both hepatitis $C$ and alcohol, 6 patients); patients having chronic hepatitis $B$ and C, 20 patients (11 patients, 9 patients respectively) and different reasons 26 patients, including cryptogenic cirrhosis in 15 patients, nonalcoholic steatohepatitis in 10 patients; Budd-Chiari syndrome in 1 patient. The etiology of liver cirrhosis is shown in Table 1.

\section{Prevalence of minimal hepatic encephalopathy}

Thirty-seven (35.9\%) patients with liver cirrhosis were found to have MHE. Out of 61 patients in CTP class A 12 (19.7\%) had MHE, 14 (51.9\%) out of 27 patients in CTP class B and $11(73.3 \%)$ out of 15 patients in CTP class $C$ had MHE $(p=0.001)$. PHES also correlated with severity of cirrhosis measured using MELD $(r=-0.345, p=0.001)$ and the CTP score $(r=-0.421, p<0.001)$ and also with ANT $(r=0.752$, $p<0.001)$. Higher CTP $(p=0.029)$ and MELD scores $(p<0.01)$ and lower albumin levels $(p=0.007)$ were observed in patients with MHE. The remaining factors such as age, etiology (alcohol vs. non-alcohol), education, hemoglobin, platelets, aspartate transaminase, alanine transaminase bilirubin, urea, creatinine and potassium levels were non-significant. Only the CTP score was found to be an independent prognostic factor for the presence of MHE in multivariable analysis. Table 3 shows the odds ratios for the variables as investigated by univariate and multivariable analyses as possible factors of minimal hepatic encephalopathy in patients with liver cirrhosis.

\section{Sensitivity and specificity of Animal Naming Test using PHES as the gold standard}

Animal Naming Test was significantly lower in patients who had cirrhosis of the liver with the presence of MHE 10.81 (95\% CI: 10.48-11.13) compared to patients without MHE 15.27 (95\% CI: 15.02-15.51, $p<0.01$ ) or as compared with controls 15.78 (95\% CI: 15.50-16.05, $p=0.002$ ) (Fig. 2). For the diagnosis of MHE based on PHES, the ROC analysis of ANT showed an areaunder-curve value of 0.978 (95\% CI: $0.954-1.0)$ (Fig. 3). At a cut-off of 14, 36 (34.9\%) patients had 
Table 3. Odds ratios for the variables as investigated by univariate and multivariable analyses as possible factors of minimal hepatic encephalopathy in patients having liver cirrhosis

\begin{tabular}{|c|c|c|c|c|}
\hline Variables & $\begin{array}{l}\text { Univariate, } \\
\text { OR (95\% Cl) }\end{array}$ & $P$-value & $\begin{array}{c}\text { Multivariable, } \\
\text { OR (95\% CI) }\end{array}$ & $P$-value \\
\hline Age (years) & $0.960(0.89-1.31)$ & 0.259 & & \\
\hline $\begin{array}{l}\text { Sex } \\
\text { (male vs. } \\
\text { female) }\end{array}$ & $2.37(0.184-5.595)$ & 0.509 & & \\
\hline $\begin{array}{l}\text { Education } \\
\text { (years) }\end{array}$ & $0.75(0.513-1.098)$ & 0.139 & & \\
\hline $\begin{array}{l}\text { Etiology } \\
\text { (alcohol vs. } \\
\text { non-alcohol) }\end{array}$ & $2.32(0.29-6.54)$ & 0.427 & & \\
\hline $\begin{array}{l}\text { Hemoglobin } \\
(\mathrm{g} / \mathrm{dl})\end{array}$ & $0.994(0.87-1.13)$ & 0.933 & & \\
\hline $\begin{array}{l}\text { Total leucocyte } \\
\text { count }\left(/ \mathrm{mm}^{3}\right)\end{array}$ & $1.0(0.99-1.0)$ & 0.233 & & \\
\hline Platelet $\left(/ \mathrm{mm}^{3}\right)$ & $1.016(1.0-1.03)$ & 0.057 & & \\
\hline Urea (mg/dl) & $1.06(0.99-1.13)$ & 0.96 & & \\
\hline $\begin{array}{l}\text { Creatinine } \\
(\mathrm{mg} / \mathrm{dl})\end{array}$ & $0.41(0.01-9.83)$ & 0.58 & & \\
\hline Sodium (mEq/l) & $0.97(0.83-1.14)$ & 0.75 & & \\
\hline Potassium (mEq/l) & $1.07(0.90-1.28)$ & 0.39 & & \\
\hline Bilirubin (g/dl) & $1.00(0.61-1.66)$ & 0.97 & & \\
\hline AST (IU/I) & $1.01(0.99-1.03)$ & 0.74 & & \\
\hline ALT (IU/I) & $0.96(0.94-0.99)$ & 0.015 & & \\
\hline Albumin (g/dl) & $2.93(2.39-3.24)$ & 0.007 & & \\
\hline ALP (IU/I) & $1.00(0.994-1.01)$ & 0.614 & & \\
\hline $\begin{array}{l}\text { Prothrombin } \\
\text { time (s) }\end{array}$ & $0.74(0.51-1.06)$ & 0.107 & & \\
\hline CTP score & $1.79(1.25-2.13)$ & 0.029 & $\begin{array}{c}1.83 \\
(1.25-2.19)\end{array}$ & 0.031 \\
\hline MELD score & $1.57(1.04-2.67)$ & 0.031 & & \\
\hline
\end{tabular}

OR - odds ratio, $\mathrm{Cl}$ - confidence interval, CTP - Child-Turcotte-Pugh, MELD - model of end stage liver disease, MHE - minimal hepatic encephalopathy, NMHE - non-minimal hepatic encephalopathy, AST - aspartate transaminase, ALT - alanine transaminase, INR - international normalized ratio

deranged ANT. Sixty-three patients had normal PHES and ANT, 33 had both abnormal PHES and ANT, 4 had normal ANT and abnormal PHES, and 3 had abnormal ANT and normal PHES (Table 4). ANT had $89 \%$ sensitivity and $95.7 \%$ specificity for the diagnosis of MHE at this cutoff if PHES is used as a reference standard; positive predictive value (PPV) was 91.6\%, negative predictive value (NPV) $94.03 \%$ and diagnostic accuracy $93.2 \%$. In the control group, ANT was $\geq 14$ in 48 out of 50 (96\%) subjects. Using the cut-off of ANT $>15$ as suggested by Amodio et al., the number

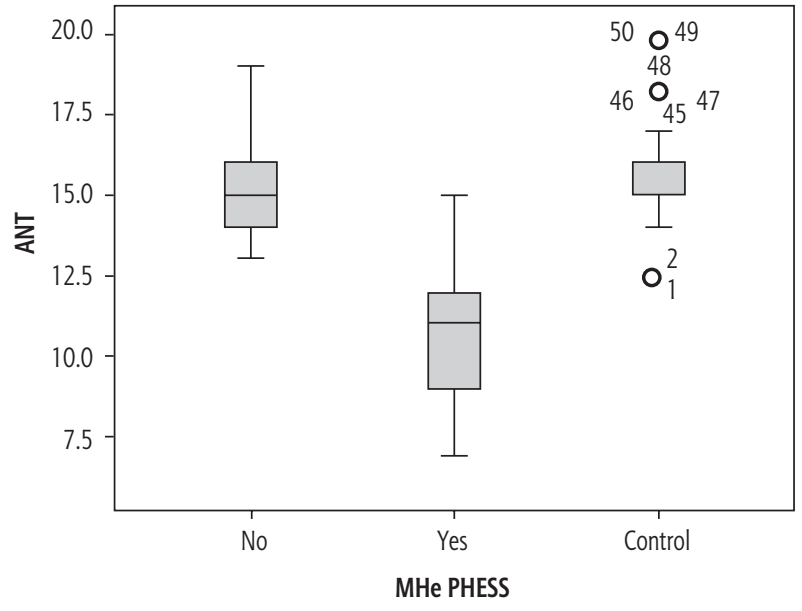

Fig. 2. ANT in controls, cirrhotics with MHE and without MHE. ANT was lower in MHE (10.81; 95\% Cl: 10.48-11.13) in comparison with non-MHE (15.27; $95 \%$ Cl: $15.02-15.51, p<0.01)$ patients having cirrhosis or controls (15.78; $95 \% \mathrm{Cl}: 15.50-16.05, p=0.002)$

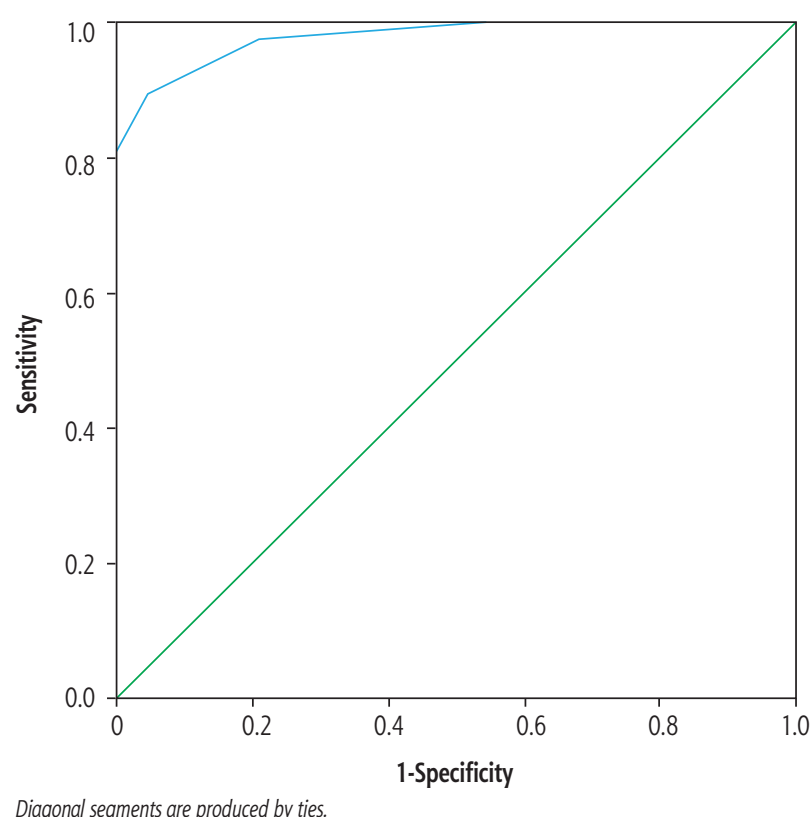

Fig. 3. Receiver operating characteristic (ROC) curve for specificity and sensitivity of ANT for diagnosis of MHE. Sensitivity was $89 \%$ and specificity was $95.7 \%$ with a cutoff of $14.0 .978(95 \% \mathrm{Cl}: 0.954-1.0)$ is the area under the curve

of healthy patients showing abnormal ANT increased to 53 (sensitivity $=97.3 \%$, specificity $=74.2 \%, \mathrm{PPV}=$ $67.9 \%, \mathrm{NPV}=98.0 \%$, accuracy $=82.52 \%)($ Table 5$)$.

\section{Factors associated with Animal Naming Test}

Animal Naming Test correlates with severity of liver disease as assessed by MELD score $(r=-0.318$, $p=0.001)$ and CTP score $(r=-0.408, p<0.001)$ in patients with liver cirrhosis. Spearman's rho correlation coefficient between ANT and the sum-score of 
Table 4. Impact of Child-Turcotte-Pugh (CTP) class, Animal Naming Test (ANT) and Psychometric Hepatic Encephalopathy Score (PHES) on risk of death

\begin{tabular}{|c|c|c|c|c|c|c|c|c|}
\hline \multirow[t]{2}{*}{ Patients with test } & \multicolumn{3}{|c|}{$\begin{array}{l}\text { CTP class A } \\
(n=61)\end{array}$} & \multicolumn{3}{|c|}{$\begin{array}{l}\text { CTP class B and C } \\
\qquad(n=42)\end{array}$} & Total & \multirow[b]{2}{*}{$\mathrm{HE}$} \\
\hline & $N$ & $n(\%)$ & $\begin{array}{l}\text { Death } \\
(n=0)\end{array}$ & HE & $n(\%)$ & $\begin{array}{l}\text { Death } \\
(n=3)\end{array}$ & $\begin{array}{l}\text { Death } \\
(n=3)\end{array}$ & \\
\hline PHES abnormal & 37 & $12(32.4 \%)$ & 0 & 4 & $25(67.6 \%)$ & 3 & 3 & 10 \\
\hline ANT abnormal & 36 & $12(33.3 \%)$ & 0 & 4 & $24(66.7 \%)$ & 3 & 3 & 10 \\
\hline PHES and ANT abnormal & 33 & $11(33.3 \%)$ & 0 & 4 & $22(66.7 \%)$ & 3 & 3 & 10 \\
\hline $\begin{array}{l}\text { PHES abnormal and ANT } \\
\text { normal }\end{array}$ & 4 & $1(25 \%)$ & 0 & 0 & $3(75 \%)$ & 0 & 0 & 0 \\
\hline $\begin{array}{l}\text { PHES normal and ANT } \\
\text { abnormal }\end{array}$ & 3 & $1(33.3 \%)$ & 0 & 0 & $2(66.7 \%)$ & 0 & 0 & 0 \\
\hline PHES and ANT normal & 63 & $48(76.2 \%)$ & 0 & 1 & $15(23.8 \%)$ & 0 & 0 & 0 \\
\hline
\end{tabular}

Table 5. Sensitivity and specificity of Animal Naming Test (ANT) for detection of minimal hepatic encephalopathy (MHE)

\begin{tabular}{lccc}
\hline Parameter & ANT - 15 & ANT - 14 & $\begin{array}{c}\text { S-ANT } \\
\text { (Campagna et al.) }\end{array}$ \\
\cline { 2 - 4 } & Value (95\% Cl) & Value (95\% Cl) & Value (95\% Cl) \\
\hline Sensitivity & $97.30 \%(85.84-99.93 \%)$ & $89.19 \%(74.58-96.67)$ & $78(70-84)$ \\
\hline Specificity & $74.24 \%(61.99-84.22 \%)$ & $95.45 \%(87.29-99.05)$ & $63(55-70)$ \\
\hline Negative predictive value (NPV) & $98.0 \%(87.58-99.71 \%)$ & $94.03 \%(86.17-97.55 \%)$ & $79(71-85)$ \\
\hline Positive predictive value (PPV) & $67.92 \%(58.35-76.20 \%)$ & $91.67 \%(78.36-97.09 \%)$ & $61(53-68)$ \\
\hline Accuracy & $82.52 \%(73.79-89.30 \%)$ & $93.20 \%(86.50-97.22 \%)$ & NA \\
\hline
\end{tabular}

$N A$ - not available

PHES was $0.652(p<0.001)$. ANT did not correlate with Handgrip $(r=0.198, p=0.045)$ in patients with cirrhosis.

\section{External validity}

All patients (66 without MHE and 37 with MHE) completed the study. All patients were on follow-up for 6 months until November 30, 2018. The mean follow-up was 244.36 days (95\% CI: 221.62-267.10), range 57-412 and inter-quartile range 166.45-354.25.

\section{Deaths}

Three patients died in total. All 3 patients had abnormal PHES and ANT on initial evaluation. All 3 deaths were in patients who had severe liver disease, i.e., CTP classes B and C (Table 3). One patient who underwent deceased donor liver transplantation (DDLT) also had severe liver disease, i.e. CTP class C. Progressive liver failure was associated with all three deaths in MHE group patients. All 3 patients developed $\mathrm{HE}$ as their terminal event.

\section{Overt hepatic encephalopathy}

During the follow-up period of 6 months 18 patients had one or more episodes of overt encephalopathy; out of these 3 developed during the terminal event causing death. Other causes were infection in 5 patients, upper gastrointestinal (UGI) bleeding in 4, diuretics in 2 and renal failure in 1 patient. Further analysis was not performed as the number of isolated episodes of overt HE was too low.

\section{Discussion}

The armamentarium of modern medical testing still has an unmet need for a rapid, easy to administer, fast and inexpensive test for the diagnosis on MHE which can be conducted by clinicians in an outpatient department. The current European Association for the Study of the Liver (EASL)/American Association for the Study of Liver Diseases (AASLD) guidelines still recommend $\mathrm{PHES}$ as a gold standard for the diagnosis of MHE [13]. However, it is time-consuming and is not being used much in clinical practice. The last two decades have seen the emergence of various other test- 
ing methods such as the Inhibitory Control Test (ICT), the Stroop EncephalApp or Critical Flicker Frequency (CFF) [5, 14-17]. However, most of these tests can only be used in specialized centres with an interest in hepatology. These tests are time-consuming, require some sort of testing equipment and are often expensive when used in the outpatient setting in the general population, which has led to the neglect of this serious complication of cirrhosis by clinicians in routine clinical practice.

In a recent study by Campagna et al. ANT was found to be a reliable and fast tool for the detection of CHE in patients with liver cirrhosis. ANT appears to be a simple, reliable test that is easy to administer in outpatient settings, with good accuracy [18]. Our study has also shown that ANT is accurate and reliable and has high specificity and sensitivity and an area under the curve for MHE diagnosis even better than that reported previously by Campagna et al.

It has been reported earlier that the prevalence of MHE among cirrhosis patients may vary from $30 \%$ to $84 \%[1,19-21]$. This study also confirms that the prevalence of MHE is high among patients with liver cirrhosis as reported previously in the same cohort with a prevalence of $40.2 \%$ [16]. It also showed that the higher CTP class is an independent predictor of the presence of MHE and the frequency of MHE also was significantly higher in patients with higher CTP scores. The different testing strategies can only partially detect patients with MHE and may not be a true reflective of cognitive dysfunction in patients with cirrhosis. The different testing methods for MHE such as EEG, PHES or ICT also have very little agreement among them for diagnosis of MHE [17, 22, 23]. However, PHES remains the gold standard for the diagnosis of MHE. In both our study and the study by Campagna et al. PHES was used as a gold standard for detection of the presence of MHE.

In our study, validation of the results for ANT as shown previously by Campagna et al. was done, and ANT was able to identify patients with MHE, patients without MHE, and healthy controls. Our results were slightly different since we divided our patient group based on education levels as $\langle 10,10-12\rangle$,12 years of education, different from the study by Campagna et al. Our study group also had a higher educational level of the patients. The reason for this is that most patients with a low level of education found it difficult to follow the instructions to complete the PHES and ANT and hence were not included in the study. Both our study and that of Campagna et al. used PHES for detection of the presence of MHE. It has been proposed that a single ANT cut-off cannot be generalized to differ- ent populations due to different education background and cultural status. So, we aimed to establish cut-off values for our patients. A cut-off of $<14$ animal names differentiated well between patients with and without MHE with a sensitivity of $89 \%$ and specificity of $95 \%$.

The different risk factors for the development of MHE as shown in previous studies were age, CTP class, portosystemic shunt, presence of oesophageal varices, and prior episodes of OHE [14]. Recent studies have also shown an association between lifestyle factors such as smoking, tobacco chewing, alcohol consumption and the presence of MHE [20]. No patients in our study had undergone portosystemic shunts or had a prior history of overt HE. However, as seen in previous studies, severity of liver disease as depicted by the CTP score was an independent predictor for the development of MHE.

MHE has an impact on the driving abilities of an individual $[24,25]$ and health-related quality of life $[1$, 26]. MHE also has an adverse effect on sleep quality of patients [27]. Impairment in cognitive functioning creates a hindrance in mental processing and attention, and hence a person's ability to make an individual decision is affected. Studies show that the administration of rifaximin or lactulose brings significant improvement in patients with MHE and the health-related quality of life and driving performance are improved $[1,17]$. Recent studies have shown that probiotics may decrease serum ammonia levels and may also improve MHE, and prevent overt HE development in patients with cirrhosis [28].

Our study also showed that MHE patients develop OHE at a significantly higher rate. $34.8 \%$ of patients in the MHE group developed OHE during the six-month follow-up period as compared to $6.1 \%$ in the patients without MHE. The results of this study were consistent with a previous study in which the incidence of developing OHE at follow-up was 3.9-17.3\% in non-MHE (NMHE) patients and 22.6-58.6\% in MHE patients [29]. The decreased rate of development of overt HE mostly is related to excluding patients with a previous history of overt $\mathrm{HE}$, and also most patients who were included in our study were in CTP classes A and B.

Survival analysis could not be done in our study since the mortality was too low to draw any strong conclusion. The reason for this low mortality might be that most patients in the study had less severe disease and belonged to CTP classes A and B. However, a key point of the study was that all patients who had death as the outcome had both altered PHES and ANT, indirectly suggesting the impact of MHE on survival.

Since currently there are no data regarding ANT in the Indian population for patients with cirrhosis, fu- 
ture studies having a larger sample size and follow-up would be required to check the reliability of ANT in our cohort and also judge whether there is a predictive ability of ANT for the development of OHE and death. Whether there is an improvement or deterioration of ANT with medical treatment is difficult to assess with the current data, and this should be investigated in further studies.

In conclusion, ANT is useful for diagnosing MHE in patients with liver cirrhosis. It is simple and accurate and may serve as a screening test in an outpatient setting before subjecting patients to more complicated paper and pencil or computerized tests.

\section{Conclusions}

Minimal hepatic encephalopathy is the mildest form in the spectrum of hepatic encephalopathy that impairs health-related quality of life.

Diagnosis of MHE is difficult in patients with cirrhosis since the presently available tests are cumbersome and time-consuming.

There is a need to develop a simple diagnostic test that can be used for the diagnosis of MHE in an outpatient setting.

The Animal Naming Test is a simple, accurate, rapid, easy-to-administer and inexpensive test for the diagnosis of MHE which can be conducted by clinicians in an outpatient department.

\section{Disclosure}

The authors report no conflict of interest.

\section{References}

1. Prasad S, Dhiman RK, Duseja A, et al. Lactulose improves cognitive functions and health-related quality of life in patients with cirrhosis who have minimal hepatic encephalopathy. Hepatology 2007; 45: 549-559.

2. Dhiman RK, Kurmi R, Thumburu KK, et al. Diagnosis and prognostic significance of minimal hepatic encephalopathy in patients with cirrhosis of liver. Dig Dis Sci 2010; 55: 2381-2390.

3. Ferenci P, Lockwood A, Mullen K, et al. Hepatic encephalopathy - definition, nomenclature, diagnosis, and quantification: final report of the Working Party at the 11th World Congresses of Gastroenterology, Vienna, 1998. Hepatology 2002; 35: 716-721.

4. Stewart CA, Smith GE. Minimal hepatic encephalopathy. Nat Clin Pract Gastroenterol Hepatol 2007; 4: 677-685.

5. Bajaj JS, Hafeezullah M, Franco J, et al. Inhibitory control test for the diagnosis of minimal hepatic encephalopathy. Gastroenterology 2008; 135: 1591-1600.e1.

6. Ridola L, Cardinale V, Riggio O. The burden of minimal hepatic encephalopathy: From diagnosis to therapeutic strategies. Ann Gastroenterol 2018; 31: 151-164.

7. Weissenborn K. Psychometric tests for diagnosing minimal hepatic encephalopathy. Metab Brain Dis 2013; 28: 227-229.
8. Romero-Gómez M, Córdoba J, Jover R, et al. Value of the critical flicker frequency in patients with minimal hepatic encephalopathy. Hepatology 2007; 45: 879-885.

9. Weissenborn K, Ennen JC, Schomerus H, et al. Neuropsychological characterization of hepatic encephalopathy. J Hepatol 2001; 34: 768-773.

10. Amodio P, Campagna F, Olianas S, et al. Detection of minimal hepatic encephalopathy: Normalization and optimization of the Psychometric Hepatic Encephalopathy Score. A neuropsychological and quantified EEG study. J Hepatol 2008; 49: 346-353.

11. Badea MA, Drug VL, Dranga M, et al. Diagnosis of minimal hepatic encephalopathy in a tertiary care center from eastern Romania: validation of the psychometric hepatic encephalopathy score (PHES). Metab Brain Dis 2016; 31: 1463-1471.

12. Dhiman RK, Saraswat VA, Verma M, et al. Figure connection test: A universal test for assessment of mental state. J Gastroenterol Hepatol 1995; 10: 14-23.

13. Vilstrup H, Amodio P, Bajaj J, et al. Hepatic encephalopathy in chronic liver disease: 2014 Practice Guideline by the American Association for the Study Of Liver Diseases and the European Association for the Study of the Liver. Hepatology 2014; 60: 715-735.

14. Kircheis G, Wettstein M, Timmermann L, et al. Critical flicker frequency for quantification of low-grade hepatic encephalopathy. Hepatology 2002; 35: 357-366.

15. Bajaj JS, Thacker LR, Heuman DM, et al. The Stroop smartphone application is a short and valid method to screen for minimal hepatic encephalopathy. Hepatology 2013; 58: 1122-1132.

16. Taneja S, Dhiman RK, Khatri A, et al. Inhibitory control test for the detection of minimal hepatic encephalopathy in patients with cirrhosis of liver. J Clin Exp Hepatol 2012; 2: 306-314.

17. Tapper EB, Parikh ND, Waljee AK, et al. Diagnosis of minimal hepatic encephalopathy: a systematic review of point-of-care diagnostic tests. Am J Gastroenterol 2018; 113: 529-538.

18. Campagna F, Montagnese S, Ridola L, et al. The animal naming test: An easy tool for the assessment of hepatic encephalopathy. Hepatology 2017; 66: 198-208.

19. Dhiman RK, Saraswat VA, Sharma BK, et al. Minimal hepatic encephalopathy: consensus statement of a working party of the Indian National Association for Study of the Liver. J Gastroenterol Hepatol 2010; 25: 1029-1041.

20. Rathi S, Chopra M, Chouduri G, et al. Prevalence of minimal hepatic encephalopathy in patients with liver cirrhosis: a cross-sectional, clinicoepidemiological, multicenter, nationwide study in India: The PREDICT study. J Clin Exp Hepatol 2019; 9: 476-483.

21. Bale A, Pai CG, Shetty S, et al. Prevalence of and factors associated with minimal hepatic encephalopathy in patients with cirrhosis of liver. J Clin Exp Hepatol 2018; 8: 156-161.

22. Montagnese S, Balistreri E, Schiff S, et al. Covert hepatic encephalopathy: agreement and predictive validity of different indices. World J Gastroenterol 2014; 20: 15756-15762.

23. Basu PP, Shah NJ. Clinical and neurologic manifestation of minimal hepatic encephalopathy and overt hepatic encephalopathy. Clin Liver Dis 2015; 19: 461-472.

24. Bajaj JS, Hafeezullah M, Hoffmann RG, et al. Navigation skill impairment: another dimension of the driving difficulties in minimal hepatic encephalopathy. Hepatology 2008; 47: 596-604.

25. Bajaj JS, Saeian K, Schubert CM, et al. Minimal hepatic encephalopathy is associated with motor vehicle crashes: The reality beyond the driving test. Hepatology 2009; 50: 1175-1183.

26. Agrawal S, Umapathy S, Dhiman RK. Minimal hepatic encephalopathy impairs quality of life. J Clin Exp Hepatol 2015; 5: S42-48. 
27. Labenz C, Baron JS, Toenges G, et al. Prospective evaluation of the impact of covert hepatic encephalopathy on quality of life and sleep in cirrhotic patients. Aliment Pharmacol Ther 2018; 48: 303-321.

28. Cao Q, Yu CB, Yang SG, et al. Effect of probiotic treatment on cirrhotic patients with minimal hepatic encephalopathy: A metaanalysis. Hepatobiliary Pancreat Dis Int 2018; 17: 9-16.

29. Saxena N, Bhatia M, Joshi YK, et al. Electrophysiological and neuropsychological tests for the diagnosis of subclinical hepatic encephalopathy and prediction of overt encephalopathy. Liver 2002; 22: 190-197. 